July $2005 \cdot \underline{\text { Volume } 99} \cdot \underline{\text { Number } 7}$

\title{
Visual Attention to Movement and Color in Children with Cortical Visual I mpairment
}

Stacey Ann Cohen-Maitre and Paul Haerich

\begin{abstract}
This study investigated the ability of color and motion to elicit and maintain visual attention in a sample of children with cortical visual impairment (CVI). It found that colorful and moving objects may be used to engage children with CVI, increase their motivation to use their residual vision, and promote visual learning.
\end{abstract}

The data presented in this article were included in the first author's doctoral dissertation. We thank the other members of the supervisory committee, Lori Annes, Kiti Freier, Hideya Koshino, and Matt Riggs, for their helpful comments and suggestions. We especially thank the participants and their parents for the time and effort that they contributed to this study. We also express our appreciation for the helpful comments and suggestions made by the editor and anonymous reviewers.

Cortical visual impairment (CVI) is one of the major forms of childhood vision loss that is encountered in the developed world (Brodskey, Baker, \& Hamed, 1996), with a prevalence as high as 72 per 100,000 children (Flanagan, Jackson, \& Hill, 2003). CVI often presents as a significant loss of visual acuity despite normal pupillary light reflexes, normal retinal morphology, and absence nystagmus (Brodskey, 1996; Good et al., 1994). Although it may 
occur as a secondary result of trauma; epilepsy; infections, such as malaria; drugs or poisons; other neurological diseases; and even a number of therapeutic procedures, it most commonly follows a perinatal hypoxic ischemic event (the reduction in blood flow and oxygen to the brain) that produces damage to the white matter of the brain near the lateral ventricles (as in periventricular leukomalacia), causes some neurons to die (apoptosis), and other neurons to differentiate abnormally (Groenendall \& Hof van Duin, 1990; Hoyt, 2003). The symptoms of CVI result when this damage affects the cortical watershed areas of the parietooccipital region or the subcortical optic radiations (Brodskey, 1996). The damage from the ischemia is often not restricted to the visual system and produces additional neurological problems, including cerebral palsy, hearing loss, and other effects that contribute to a general developmental delay.

Although diffuse cerebral atrophy, white matter hyperintensities, and periventricular leukomalacia are often observed in CT (computerized tomography) and MR (magnetic resonance) images (Baker-Nobles \& Rutherford, 1995; Brodskey et al., 1996; Jan, Groenveld, \& Andersen, 1993), some visual function is often preserved. In one study, less than $10 \%$ of the children had no clinically measurable visual response (Flanagan et al., 2003); the remainder displayed various levels of response to light and visually presented objects. Furthermore, the amount of residual visual ability has been considered to be predictive of functional improvement. For example, visual evoked potentials (VEPs) that were elicited in children with CVI, although abnormal in form and time course, were indicative of a positive prognosis (BakerNobles \& Rutherford, 1995). In this context, then, the term cortical visual impairment has been used instead of cortical blindness to emphasize the preservation of some degree of visual capacity in the majority of these children (Hoyt, 2003).

\section{Visual responses in CVI}


Behavioral observations have been considered the optimal method of assessing the preservation of function in the visual system in children with CVI, many of whom are preverbal or have limited verbal abilities (Jan et al., 1993). A large proportion of these children exhibit light gazing (staring for long periods at a light source) (Baker-Nobles \& Rutherford, 1995; Jan, Groenveld, \& Sykanda, 1990), and the majority of children with CVI who do not light gaze display photophobia (Jan et al., 1993; Jan \& Wong, 1991). In both cases, the children display a behaviorally obvious sensitivity to the presence of a light stimulus. Moreover, many children with CVI not only respond to visual stimulation, but have some response to specific stimuli in the environment (Flanagan et al., 2003). We were particularly interested in two classes of visual sensitivity that are observed in most children with CVI: the perception of color and of motion.

Clinical reports have indicated that many children with CVI have near-normal color vision, probably because color is abundantly represented bilaterally in the visual cortex, and color-specific cortical areas (such as V4) are often relatively spared. With the cortical (or subcortical) damage resulting in a "Swiss cheese-like" visual field containing multiple scotomas, color information is more likely to be processed with some degree of success, especially compared with the perception of form (Good et al., 1994). Thus, it is not surprising that many children with CVI can respond to a variety of colors and can even be taught to name colors or associate specific colors with objects or shapes (BakerNobles \& Rutherford, 1995; Jan, Groenveld, Sykanda, \& Hoyt, 1987; Jan \& Wong, 1991; Merrill \& Kewman, 1986). Not only is color vision often preserved, but some reports have suggested that a preference for bright colors, such as red or yellow, is common among children with CVI (Baker-Nobles \& Rutherford, 1995; Jan et al., 1987; Jan \& Wong, 1991). However, clinical observations by the first author suggested that although individual children 
may demonstrate an idiosyncratic color preference, the preference is not readily predicted by a diagnosis of CVI.

The perception of motion is another parameter of visual processing that is often spared in CVI. Whether because of the preservation of the retino-collicular pathways or the intact function of some threshold amount of the visual fields, children with CVI can often detect movement and, when ambulatory, avoid obstacles (Benton, Levi, \& Swash, 1980; Jan et al., 1987).

To examine the visual competencies that are available to children with CVI and their therapists, we conducted a series of experiments to investigate the perceptual responsiveness to color and movement of a sample of children with CVI. In particular, we investigated the ability of these visual features to elicit and hold visual attention on the basis of the rationale that such stimuli would be most useful for education, treatment, and remediation. We wanted to know if color would have an advantage in producing a response over luminant stimuli in general; if any particular color was commonly preferred; and, given that movement was effective, if color would add to the preference for a moving stimulus.

In these experiments, pairs of visual stimuli were presented, and the children's preferential gaze was observed. Two video monitors, placed about 2 meters (about 6.5 feet) apart, displayed visual patterns consisting of a horizontal bar on a solid background. The bar could be gray, green, blue, red or yellow. It could be stationary or appear to move upward and downward. Each experiment compared specific aspects of these features and assessed which was more effective in eliciting visual attention. Across the experiments, we compared each color against a gray of equivalent luminance, each color against the other colors, and various combinations of moving and static stimuli. We expected that if the subject's cortical visual system were sufficiently intact 
to process colors, then colors would be more effective in attracting gaze than would a gray stimulus of equivalent luminance. Furthermore, if one color was preferred over the others, it would become apparent as a preference in gaze. We expected that movement would be a particularly effective feature for attracting gaze. However, we could not predict the relative strength of movement in comparison with color in attracting and holding visual attention.

\section{General method}

\section{Participants}

Eleven children aged 18-72 months with a diagnosis of CVI, a codiagnosis of cerebral palsy (CP), no other neurological or psychiatric diagnoses, and sufficient visual and motor ability to participate in a forced-choice preferential looking task were recruited for this study. Each of their parents signed a release form allowing the child's neuro-ophthalmologist to confirm the CVI diagnosis. Data from 2 participants were omitted from the analyses because of difficulty in consistently coding their eye movements. The 9 remaining participants included 4 boys and 5 girls, who ranged in age from 28 to 67 months. The parents reported that 7 of the 9 children appeared to prefer visual stimulation in their right visual fields.

The parents completed the Functional Skills Questionnaire, which provides information about a child's gross motor and sensorimotor status (Birch \& Bane, 1991). Overall, there was significant impairment in both gross motor and sensorimotor functioning (with mean scores of 5.4 out of a possible 11, and 6.2 out of a possible 14, respectively). One participant displayed no motor impairment (scores of 11 and 14). However, his visual performance was similar to that of the other participants. 
All the procedures for this study were approved by the local institutional review boards. Each set of parents provided informed consent for their child's participation.

\section{Stimuli and apparatus}

The experiment was conducted in a small testing office without windows. Stimuli were presented on two 19-inch SONY Trinitron color monitors that were placed at a 2-meter (about 6.5-foot) viewing distance from the child and separated at a 15-degree visual angle, measured center to center. A Minolta Spot meter was used to match the luminance of the two displays. A black curtain was placed between the two monitors. An opening in the curtain allowed the positioning of two SONY VX-1000 digital video cameras, which were used to record the child's eye and head movements, respectively.

Stimuli were generated and presented by a single 486-based personal computer that was interfaced with the two monitors. The stimulus consisted of horizontal bars, 23 centimeters (about 9 inches) in width, presented in yellow, green, blue, red, or gray against a black background. Each color was presented in four levels of luminance and was matched to a gray stimulus of equivalent luminance (RGB codes for generating the color stimuli that were used in this study are available from us on request).

Bars were presented either as static figures or moving in an upward or downward direction at one of four velocities (3.5, 7, 14, or 21 pixels per second). Although infants may be more likely to scan vertical than horizontal contours, we used horizontal bars in this study because the vertical bars appeared to jitter or vibrate when they moved (Haith, 1980; Kessen, Salapatek, \& Haith, 1972). The output of each of the two monitors and the two digital cameras was directed through a quad-splitter, combined with a time code, and recorded on videotape for offline scoring and analysis. 


\section{Procedure}

Each child was seated in his or her own wheelchair or in a stationary chair at eye level with the center of the two monitors at a 2-meter (about 6.5-foot) distance. The child's parent or caretaker was seated in a chair directly behind the child to provide care and support, as needed, during the session. Each parent or caretaker was instructed to refrain from interacting with the child during the trials. It was necessary for one child to be held in the parent's lap; this parent was asked to wear a blindfold during the study.

We verified each child's ability to gaze directly toward each of the two monitors by alternately presenting a moving stimulus on one screen or the other (Birch \& Bane, 1991). All the participants were able to gaze directly toward the monitor that was presenting a stimulus and subsequently to shift their gaze to the other monitor following the stimulus.

At the start of each trial, a small, blue, green, red, and yellow multicolored, rotating light, which was positioned at the midline between the two monitors, was illuminated. This light was used to attract the child's fixation to the center for the start of the trial. The light stimulus was supplemented with auditory stimuli (squeaky toys and music boxes), as needed, to attract the child's attention. The experimenter allowed the trial to begin only when the child's attention was directed to the center. Once presented, the stimuli remained visible for 10 seconds. The child's gaze was scored offline for the direction and duration of the initial look and the total number and total duration of looks at each stimulus.

A break was provided between each experiment, roughly every 16 to 32 trials. In addition, a child was allowed to take a break if he or she appeared to be restless or fussy. The entire test session took 50 to 60 minutes, depending on the number and duration of 
breaks that the child required to maintain an alert and attentive state. Nevertheless, not all the children completed the entire set of seven experiments.

\section{Scoring and analysis}

The video record of each trial was scored by two trained observers, both of whom were familiar with the visual behaviors of children with CVI but were not aware of the position and content of the visual displays. Using a video recorder with frameby-frame playback capability, the observers scored each trial for the duration and number of gaze fixations at each monitor (the results for the dependent measures - initial fixation duration, total number of gaze fixations, and total duration of gaze fixationsare available from us on request). The reliability of the two raters was high. The intraclass correlations for the duration of both total fixation and initial fixation ranged from .94 to .97 across the participants. For the number of fixations, the intraclass correlations ranged from .90 to .99.

The analytic strategy focused on the major factors of color and whether or not the stimulus was moving. When possible, analyses of variance (ANOVAs) were conducted on each of three dependent variables: the mean duration of the initial gaze fixation, the mean number of fixations during the 10 -second trial, and the mean total gaze duration across the trial. However, because the number of trials was necessarily small to maintain an alert and attentive state in the children, it was often impossible to conduct a complete ANOVA. The factors of velocity, luminance, or direction of movement were omitted in all the analyses.

Moreover, since some children were unable to complete all the trials of a given experiment, planned ANOVAs across colors were reduced to paired comparisons. Furthermore, the small number of participants reduced the power of the analyses, resulting in the likelihood of observing only the largest effects. 


\section{Experimental design and results}

The seven experiments proceeded in a fixed order with a twominute break between each. Within each experiment, the trials were presented in a random order, determined by the computer.

\section{Experiment 1: Color-gray}

In each trial, a color stimulus (yellow, green, blue, or red) appeared on one monitor while the luminance-matched gray stimulus appeared simultaneously on the other monitor. Neither stimulus was a moving stimulus. A factorial combination of color (4 levels), luminance (4 levels), and monitor (left, right) produced 32 trials.

Although no preference was found in the duration of their initial gaze, $t(7)=2.22, p=.06$, or in the number of gazes, $t(7)<1, p=$ $n s$, the children spent more time looking at colors than at luminance-matched gray, $t$ (7) 3.39, $p<.01$. As may be seen in Figure 1, although the preference for color over gray is clear, there was no significant preference among the colors.

\section{Experiment 2: Color-color}

In each trial, a color stimulus appeared on one monitor while another color stimulus was presented simultaneously on the other monitor. Only the two intermediate luminance levels were used for this experiment. Neither stimulus was a moving stimulus. The color pairs were matched for luminance. A factorial combination of color (4 levels), luminance (2 levels), and monitor (left, right) produced 16 trials.

Across the three measures, no preference was observed among any of the colors, $F(3,21)<1.65, p>.10$ (see Figure 2). Because colors were paired with colors in all the trials, we were able to 
examine lateral bias as the effect of presenting colors simultaneously in the left or right visual fields. The main effect of visual field was not significant in any of the analyses, $F(1,7)<$ $3.68, p>$.10. None of the interactions was significant.

\section{Experiment 3: Gray-gray movement}

In each trial, a nonmoving gray stimulus appeared on one monitor while a luminance-matched gray moving stimulus was presented simultaneously on the other monitor. A factorial combination of luminance (4 levels), velocity (4 levels), and monitor (left, right) produced 32 trials. The direction of movement (up, down) varied randomly across trials but was not explicitly balanced.

In the comparison of a moving gray bar with a static gray stimulus, movement produced a strong effect that led to a longer initial fixation, more frequent subsequent fixations during the trial, and a longer total fixation time, $t(8)>2.91, p<.02$ (see Figure 3).

\section{Experiment 4: Color-color movement}

In each trial, a nonmoving color stimulus appeared on one monitor while a luminance-matched moving stimulus in the same color was presented simultaneously on the other monitor. A factorial combination of color (4 levels), velocity (4 levels), and monitor (left, right) produced 32 trials. The direction of movement (up, down) varied randomly across the trials but was not explicitly balanced.

As with the gray stimuli in the previous experiment, movement produced a strong effect that led to a longer initial fixation, more frequent subsequent fixations during the trial, and a longer total fixation time, $t(8)>4.06, p<.01$ (see Figure 4). There were no apparent preferences among the colors. 


\section{Experiment 5: Color-gray movement}

In each trial, a static color stimulus appeared on one monitor while a luminance-matched moving gray stimulus was presented simultaneously on the other monitor. A factorial combination of color (4 levels), velocity (14 and 21 pixels per second), direction (up, down), and monitor (left, right) produced 32 trials.

Once again, movement produced a strong effect that led to more frequent fixations during the trial and a longer total fixation time, $t(8)>9.45, p<.01$ (see Figure 5). However, color also

demonstrated some attention-attracting quality, since the duration of the initial fixation to color ( $M=3.5$ seconds) was longer than was the duration of the initial fixation to the moving gray stimulus ( $M=0.8$ seconds), $t(8)=4.45, p<.01$. There were no apparent preferences among the colors.

\section{Experiment 6: Color movement-gray movement}

In each trial, a moving color stimulus appeared on one monitor while a luminance-matched moving gray stimulus was presented simultaneously on the other monitor. As in Experiments 2 and 5, respectively, only the two intermediate levels of luminance and the 14- and 21 pixels-per-second velocities were used. A factorial combination of color (4 levels), luminance (2 levels), velocity (2 levels), and monitor (right, left) produced 32 trials.

When both stimuli were moving, color provided an additive effect that led to a longer initial fixation, more frequent subsequent fixations during the trial, and a longer total fixation time, $t(7)>$ $3.12, p<.02$ (see Figure 6). There were no apparent preferences among the colors.

\section{Experiment 7: Color on a black background-black on a color background}


In each trial, a nonmoving color stimulus appeared on one monitor while a stimulus with the bar and background colors reversed was presented simultaneously on the other monitor. Thus, a red bar on a black background was paired with a black bar on a red background. In addition, yellow, green, blue, and white bars (or backgrounds) were included in these trials. A factorial combination of color (5 levels) and monitor (left, right) produced 10 trials, each of which was repeated in a second block for a total of 20 trials.

No differences were observed in the gaze of the children when they viewed color that was presented against a black background compared to when they viewed black that was presented against a color background, $t(6)<1.21, p>.10$ (see Figure 7).

Furthermore, there were no apparent preferences among the colors.

\section{Discussion}

It is clear from this series of experiments that not only were basic color and motion perception preserved in this sample of children with CVI, but color and movement are visual features with salient, independent, and additive effects on visual attention. The first primary research question was whether color has an attentional advantage over luminant stimuli in general. The color displays attracted and maintained attention more effectively than did the gray displays in Experiments 1, 5, and 6. The second primary research question concerned the ability of movement to attract and hold visual attention. Experiments 3, 4, and 5 demonstrated that moving displays drew more attentional focus than did static displays. Moreover, the separate and independent attraction for attentional focus that was exerted by color was observed in Experiment 6. Despite the ability of movement to capture attention, the moving color bar was preferred to an 
equally luminant moving gray bar.

In comparing the relative salience of movement and color, we found that movement is more effective in drawing visual attention. The color stimulus was preferred to a gray stimulus of equal luminance (Experiment 1). Nevertheless, in each experiment in which a moving stimulus was paired with a static one (Experiments 3, 4, and 5), motion attracted the children's gaze and held it for a greater duration. This preference for movement held for gray stimuli and for color stimuli. Even when the static stimulus was color, the children preferentially directed their gaze to a gray bar in motion.

The results of this study underscore the observations of physicians, therapists, and parents that children with CVI exhibit strong visual preferences for color and for moving objects (BakerNobles \& Rutherford, 1995; Good et al., 1994; Jan et al., 1987; Jan \& Wong, 1991). However, these experiments also challenge the reports that children with CVI show a consistent preference for red or yellow compared with other colors (Good et al., 1994). A preference for specific colors was the primary issue in Experiment 2. That experiment revealed no consistent preference for any of the colors. An examination of the preferences displayed in Experiments 1 and 6 suggests that blue and yellow elicited the greatest preference over the gray stimuli when the stimulus pairs were static and moving, respectively (Figures 1 and 6). However, none of these comparisons was statistically reliable. Nevertheless, it must be remembered that the small sample in this study limited our ability to detect a color preference. It is possible that the children in this study did have color preferences, as has been previously described. If so, it may mean that the size of the colorpreference effect is relatively small in comparison to general preferences for color or motion. Additional data need to be collected across a larger sample of children with CVI to examine the issue of generalizability for this as well as all our other results. 
It may be noted that all our stimuli were, in a technical sense, color stimuli. Because we used color monitors to present the stimuli, the color and gray displays differed only in the relative luminance of the red, green, and blue components of which the displays are composed. However, because the color displays were not produced simply by setting one component of the monitor display high and the other two low (for example, red was produced by RGB codes 233, 144, 146 and 255, 212, 217), a preference for color displays over gray is unlikely to reflect merely the relative increase in luminance of one compared to the other two component channels of the monitor displays. Because the overall luminance of the displays was controlled, we believe that these data are better interpreted as a preference for color than for high-luminance displays.

We were not able to find previous reports that indicated a preference for motion over color. To the contrary, the anecdotal literature and the design of some commercially available "light boxes" assume that color is more important than motion and that attention is maximally engaged by color that is presented on black backgrounds. As we noted earlier, these experiments indicate that motion is more effective than color in attracting visual attention, although the effects are independent and additive. Moreover, although we presented a color (or gray) stimulus against a black background for most of the experiments, Experiment 7 manipulated the use of color for the background or foreground and demonstrated no effect on the orientation of visual attention.

\section{I mplications for practice}

Although the findings do not provide direct support for the specific design of light boxes, the results may be useful in designing and implementing therapies for children with CVI. They suggest that both color and movement may be used in 
working with children with CVI. For example, when a child's color vision appears to be intact, a parent or therapist can ensure that each object that is used in a given context maintains a consistent color. Thus, the use of a red plate combined with a blue cup for meals, along with the avoidance of other brightly colored items, may help the child distinguish the plate and cup, despite the absence of effective form perception. The goal would be to facilitate interaction with the environment. For example, as the child becomes familiar with and develops associations between colors and functions (such as blue for a cup, red for a plate, and yellow for a spoon), locating and reaching for the object will become easier (see Farrenkopf, McGregor, Nes, \& Koenig, 1997).

In addition, color and motion may be combined to help teach children with CVI basic concepts, such as speed, depth, and distance. For example, using a lazy Susan, a teacher or parent could spin a brightly colored object at fast and slow velocities to teach the concept of speed. By moving the object to the front and rear positions, the concepts of near and far could be demonstrated. Similarly, by having one object move relative to another, different-color objects "in front of" and "behind" could be displayed.

Consistency and simplicity are important principles for using color and motion to help children with CVI understand their visual environment. In any given interaction, the number of important objects should be kept to a minimum. Care should also be taken not to clutter the home with too many colorful objects. Multiple objects of the same color could lead to confusion, particularly if the location of the objects is inconsistent. Storing objects in a consistent location or moving them in consistent ways may allow a child to become familiar with the environment and, in turn, facilitate interactions with his or her caregiver and others by allowing the child to lead the caregiver or orient toward the object of interest. Furthermore, it is important to allow the child 
time to respond; children with CVI often require additional time to process visual information and to generate a response.

Moreover, physicians; occupational-, physical-, speech and language-, and other therapists; psychologists; and teachers may benefit from recognizing the impact of color and motion in their assessment, evaluation, and treatment of children with CVI. For example, using large, colorful, moving objects or encouraging the child to move whenever possible may increase the child's motivation during an evaluation and provide a more accurate estimation of the child's visual function and competence.

Finally, we have noticed that the characteristics of visual function in children with CVI are similar to those of some adults who are diagnosed with visual agnosia (inability to recognize or attach meaning to something that is perceived). Often these individuals show focal cortical damage to the parieto-occipital areas with relative sparing of the primary visual cortices, which leads to multiple scotomas and "Swiss Cheese"-like visual fields. Using the taxonomy of visual agnosias developed by Farah (2004), we think that most children with CVI appear to fall within the narrow sense of apperceptive agnosia or dorsal simultanagnosia. Like children with CVI, adults with apperceptive agnosia tend to have adequate elementary visual functions for the perception of color and motion, but their ability to discriminate form or shape is impaired. These adults have difficulty identifying an object from its visual features alone. Rather, they identify an object by combining basic visual information, such as color, with information from their other sensory modalities. Thus, sound, texture, and/or smell are critical to the process of identifying objects for both children with CVI and adults with apperceptive agnosia. Moreover, like some children with CVI, adults with dorsal simultanagnosia have a compromised but not absent ability to recognize objects. Their form perception is poor, and they recognize only some part of an object, not the whole. 
Given the similarities in both the observed symptoms and the affected brain regions, we suggest that an additional change in terminology may be appropriate - that CVI should be referred to as developmental visual agnosia (DVA). As with acquired visual agnosia, subtypes of DVA may be distinguished: an apperceptive type and a simultanagnosic type. In addition, the onset of symptoms may be specified as early or late to indicate whether a child experienced a period of normal visual development after birth. This change in terminology would not only emphasize the preservation of some visual function (as in the comparison of CVI with cortical blindness), but would facilitate communication, consultation, and collaboration among the medical and neuropsychological communities.

\section{Summary}

In summary, this study was the first to demonstrate empirically the importance of both color and motion in the visual attention of children with CVI. The results were consistent with some previous observations in demonstrating the importance of color perceptions. In addition, the attention-attracting power of motion was observed. These results may be applied in educational, therapeutic, and home environments as interventions, since the use of colorful and/or moving objects is likely to engage children with CVI and increase their motivation to use their residual vision and, in turn, promote their efficiency in visual learning.

\section{References}

Baker-Nobles, L., \& Rutherford, A. (1995). Understanding cortical visual impairment in children. American Journal of Occupational Therapists, 49, 899-903.

Benton, S., Levi, I., \& Swash, M. (1980). Vision in the temporal 
crescent in occipital infarction. Brain, 103, 83-97.

Birch, E. E., \& Bane, M. C. (1991). Forced-choice preferential looking acuity of children with cortical visual impairment. Developmental Medicine and Child Neurology, 33, 722-729.

Brodskey, M. C. (1996). The apparently blind infant. In M. C. Brodskey, R. S. Baker, \& L. M. Hamed (Eds.), Pediatric neuroophthalmology (pp. 11-41). New York: Springer Verlag.

Brodskey, M. C., Baker, R. S., \& Hamed, L. M. (Eds.). (1996). Pediatric neuro-ophthalmology. New York: Springer Verlag.

Farah, M. J. (2004). Visual agnosia (2nd ed.). Cambridge, MA: MIT Press.

Farrenkopf, C., McGregor, D., Nes, S. L., \& Koenig, A. J. (1997). Increasing a functional skill for an adolescent with cortical visual impairment. Journal of Visual Impairment \& Blindness, 91, 484-493.

Flanagan, N. M., Jackson, A. J., \& Hill, A. E. (2003). Visual impairment in childhood: Insights from a community-based survey. Child: Care, Health \& Development, 29, 493-499.

Good, W. V., Jan, J. E., DeSa, L., Barkovich, A. J., Groenveld, M., \& Hoyt, C. S. (1994). Cortical visual impairment in children. Survey of Ophthalmology, 38, 351-364.

Groenendall, F., \& Hof van Duin, J. V. (1990). Partial visual recovery in two full-term infants after perinatal hypoxia. Neuropediatrics, 21, 76-78.

Haith, M. (1980). Rules that babies look by. Hillsdale, NJ: Lawrence Erlbaum. 
Hoyt, C. S. (2003). Visual function in the brain-damaged child. Eye, 17, 369-384.

Jan, J. E., Groenveld, M., \& Andersen, D. P. (1993).

Photophobia and cortical visual impairment. Developmental Medicine and Child Neurology, 35, 473-477.

Jan, J. E., Groenveld, M., \& Sykanda, A. M. (1990). Light gazing by visually impaired children. Developmental Medicine and Child Neurology, 32, 755-759.

Jan, J. E., Groenveld, M., Sykanda, A. M., \& Hoyt, C. S. (1987). Behavioral characteristics of children with cortical visual impairment. Developmental Medicine and Child Neurology, 21, 571-579.

Jan, J. E., \& Wong, P. K. H. (1991). The child with cortical visual impairment. Seminars in Ophthalmology, 6, 194-200.

Kessen, W., Salapatek, P., \& Haith, M. (1972). The visual response of the human newborn to linear contour. Journal of Experimental Child Psychology, 13, 9-20.

Merrill, M. K., \& Kewman, D. G. (1986). Training of color and form identification in cortical blindness: A case study. Archives of Physical Medicine and Rehabilitation, 67, 479-483.

Stacey Ann Cohen-Maitre, Ph.D., psychologist, Verdugo Hills Autism Project, 16530 Ventura Boulevard, Encino, CA, 91436; email: < msmaitre@aol.com>.Paul Haerich,Ph.D., professor, Brain Studies and Neurocognition, Department of Psychology, Loma Linda University, Loma Linda, CA, 92350; e-mail: <phaerich@psych.llu.edu>.

$\underline{\text { Previous Article } \mid \text { Next Article } \mid \text { Table of Contents }}$ 
JVIB, Copyright (C 2005 American Foundation for the Blind. All rights reserved.

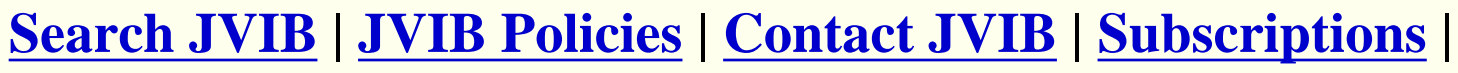 JVIB Home}

If you would like to give us feedback, please contact us at jvib@afb.net.

\section{www.afb.org | Change Colors and Text Size | Contact Us | Site Map | Site Search Go

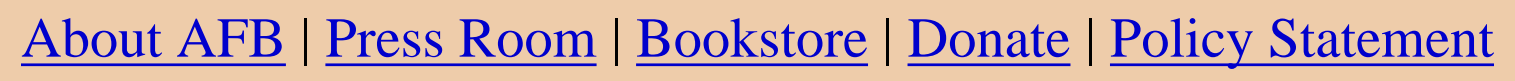

Please direct your comments and suggestions to afbinfo@afb.net Copyright (c) 2005 American Foundation for the Blind. All rights reserved. 